\title{
SOME THEOREMS ON HOLONOMY GROUPS OF RIEMANNIAN MANIFOLDS
}

\author{
BY \\ SHIGEO SASAKI AND MORIKUNI GOTO
}

For Riemannian manifolds there are four kinds of holonomy groups: the (nonrestricted) holonomy group $H$, the restricted holonomy group $H^{0}$, the (nonrestricted) homogeneous holonomy group $h$, and the restricted homogeneous holonomy group $h^{0}$. It is known that all of these are Lie groups of transformations and $H^{0}$ and $h^{0}$ are the connected components of the identity of $H$ and $h$ respectively.

1. Relations among invariant linear subspaces of $h^{0}$ and $h$. If the restricted homogeneous holonomy group $h^{0}$ is reducible (in the real number field) it is completely reducible, for $h$ is a subgroup of the orthogonal group. If the holonomy group $h^{0}$ is reducible, we can take a repère in the tangent space $E_{n}(O)$ at the base point $O$ of the holonomy group so that all elements of the group $h^{0}$ can be represented by matrices of the following type:

$$
T=\left(\begin{array}{llll}
{ }^{T_{1}} & & & 0 \\
& T_{2} & & \\
& & \cdot & \\
& & & T_{m}
\end{array}\right) .
$$

We assume that the group of matrices $T_{\lambda}$ is irreducible for each $\lambda(\lambda=1, \cdots$, $m)$. Let us denote the linear vector space on which $T_{\lambda}$ operates by $E_{(\lambda)}$, $E_{(\lambda)}$ 's are called irreducible invariant linear subspaces. If $T_{m}$ is of dimension 1 , $T_{m}$ is equal to 1 .

In the same way we can consider the reducibility of the group $h$. It may happen that, for example, $E_{(1)}$ is not invariant under $h$ although it is invariant under $h^{0}$. In such a case there exists a closed curve $C_{\alpha}$ of class $D^{\prime}$ passing through the base point 0 of our holonomy groups such that the congruent transformation $T\left(C_{\alpha}\right)$ associated with it takes $E_{(1)}$ into another linear subspace $E_{(1) \alpha}$ :

$$
T\left(C_{\alpha}\right) E_{(1)}=E_{(1) \alpha} .
$$

We shall denote the element of the fundamental group $\pi_{1}$ to which $C_{\alpha}$ belongs by $\alpha$.

If we take another closed curve $C_{\alpha}^{\prime}$ passing through 0 , the product curve $C_{\alpha}^{\prime} C_{\alpha}^{-1}=C_{0}$ is homotopic to zero. Hence we get

$$
T\left(C_{0}\right)=T\left(C_{\alpha^{-1}}^{-1} T\left(C_{\alpha}^{\prime}\right) .\right.
$$

As $T\left(C_{\alpha}^{-1}\right)=T\left(C_{\alpha}\right)^{-1}$, we see

$$
T\left(C_{\alpha}^{\prime}\right)=T\left(C_{\alpha}\right) T\left(C_{0}\right),
$$

Received by the editors April 6, 1954 and, in revised form, November 15, 1954. 
and accordingly we get

$$
T\left(C_{\alpha}^{\prime}\right) E_{(1)}=T\left(C_{\alpha}\right) T\left(C_{0}\right) E_{(1)}=T\left(C_{\alpha}\right) E_{(1)}=E_{(1) \alpha \cdot}
$$

Therefore the transformation $T$ associated to any closed curve passing through $O$ and belonging to $\alpha$ takes $E_{(1)}$ to the same $E_{(1) \alpha}$.

In the next place, we can show that the linear subspace $E_{(1) \alpha}$ is invariant under the group $h^{0}$. To prove this, let us take an arbitrary closed curve $C_{0}$ of class $D^{\prime}$ passing through $O$ and homotopic to zero. Then the product curve $C_{\alpha} C_{0}$ is homotopic to $C_{\alpha}$, whence by virtue of the above result we get

$$
T\left(C_{0}\right) T\left(C_{\alpha}\right) E_{(1)}=E_{(1) \alpha},
$$

whence

$$
T\left(C_{0}\right) E_{(1) \alpha}=E_{(1) \alpha}
$$

which is to be proved.

In the same way it may happen that transformations associated to closed curves passing through $O$ and belonging to an element $\beta$ of $\pi_{1}$ take $E_{(1)}$ to a linear subspace $E_{(1) \beta}$ different from $E_{(1)}$ and $E_{(1) \alpha}$. It is easily seen that transformations associated to closed curves passing through $O$ and belonging to the element $\alpha^{-1} \beta$ take $E_{(1) \alpha}$ to $E_{(1) \beta}$.

We shall assume that $\operatorname{dim}\left(E_{(1)}\right) \geqq 2$. Then $E_{(1) \alpha}$ must coincide with one of $E_{(2)}, \cdots, E_{(m)}$.

To show this let us take a vector $v$ of $E_{(1) \alpha}$, then it can be written in the following form:

$$
v=v_{1}+v_{2}+\cdots+v_{m},
$$

where $v_{\lambda} \in E_{(\lambda)}$. First we assume that $v_{1} \neq 0$. According to Borel-Lichnerowicz's theorem( $\left.{ }^{1}\right)$ we know that the restricted group $h^{0}$ is a direct product of component groups of matrices: $h^{0}=h_{(1)}^{0} \times h_{(2)}^{0} \times \cdots \times h_{(m)}^{0}$. Hence $h^{0}$ contains the group $h_{(1)}^{0} \times 1 \times \cdots \times 1$ as its subgroup. If we denote a transformation of this group by $T$, we get

$$
T(v)-v=T\left(v_{1}\right)-v_{1},
$$

the last vector belongs to $E_{(1)} \cap E_{(1) \alpha}$. As $h_{(1)}^{0}$ is irreducible there exists a $T$ such that $T\left(v_{1}\right)-v_{1}$ is not the null vector. This contradicts the fact that $E_{(1)} \neq E_{(1) \alpha}$ and $E_{(1)}$ is irreducible. Accordingly $v_{1}$ must be equal to zero.

By the same argument, we can see that if $\operatorname{dim}\left(E_{(\lambda)}\right) \geqq 2 \lambda$ fixed), then either $v_{\lambda} \neq 0$ (other $v_{\mu}=0, \mu \neq \lambda$ ) and $E_{(1) \alpha}$ coincides with $E_{(\lambda)}$ or $v_{\lambda}=0$. As $E_{(1) \alpha}$ does

(1) A. Borel and A. Lichnerowicz, Groupes d'holonomie des varittes riemanniennes, C. R. Acad. Sci. Paris vol. 234 (1952) pp. 1835-1837. 
not contain invariant vectors, this proves that $E_{(1) \alpha}$ coincides with one of $E_{(2)}, \cdots, E_{(m)}$. Q.E.D.

We shall change the notation if it is necessary and can assume that $E_{(1) \alpha}$ coincides with $E_{(2)}$.

In the same way, if $E_{(1) \beta}$ is different from $E_{(1)}$ and $E_{(2)}$ it coincides with one of $E_{(3)}, \cdots, E_{(m)}$. We can assume that $E_{(1) \beta}$ coincides with $E_{(3)}$.

Repeating this process we can see that there exists a minimal set of linear subspaces $E_{(1)}, \cdots, E_{(k)}$ such that they are transformed to each other by the holonomy group $h$ and no other linear subspaces are obtained from them by $h$. Then the direct sum of these spaces constitutes an irreducible invariant subspace of $h$.

Consequently, we get the following theorem:

THEOREM 1. Suppose that the restricted homogeneous holonomy group $h^{0}$ is reducible and let $E_{(1)}, \cdots, E_{(m)}$ be irreducible invariant subspaces. If $E_{(1)}$ is not invariant under $h$ and $\operatorname{dim} E_{(1)} \geqq 2$, we consider the irreducible invariant subspace under $h$ which contains $E_{(1)}$ and denote it by $E_{(1)}^{*}$. Then we can select, from $E_{(1)}, \cdots, E_{(m)}, l_{1}\left(l_{1}=\operatorname{dim} E_{(1)}^{*} / \operatorname{dim} E_{(1)}\right)$ linear subspaces all of the same dimension such that they span $E_{(1)}^{*}$ and each of them can be transformed from any other of them by some transformations of $h$.

We change the notation if it is necessary and can assume that these $l_{1}$ linear subspaces are $E_{(1)}, \cdots, E_{\left(l_{1}\right)}$. If $\operatorname{dim} E_{\left(l_{1}+1\right)} \geqq 2$ and $E_{\left(l_{1}+1\right)}$ is not invariant under $h$, then we can consider the irreducible invariant subspace under $h$ which contains $E_{\left(l_{1}+1\right)}$. We denote it by $E_{(2)}^{*}$ and assume that $E_{(2)}^{*}$ consists of $E_{\left(l_{1}+1\right)}, \cdots, E_{\left(l_{1}+l_{2}\right)}$, and so on.

If there are one-dimensional subspaces among $E_{(1)}, \cdots, E_{(m)}$, we collect them altogether at the last part of the sequence of subspaces. Suppose that $E^{(1)}$ is one of the subspaces $E_{(1)}, \cdots, E_{(m)}$ and such that $\operatorname{dim} E^{(1)}=1$. If $E^{(1)}$ is not invariant under $h$, then as before there exist vectors $E^{(1) \alpha}, E^{(1) \beta}, \ldots$ invar iant under $h^{0}$ and not equal to $E^{(1)}$ and derived from $E^{(1)}$ by some transformations of $h$. However, contrary to the former case, we cannot say that $E^{(1)}, E^{(1) \alpha}, E^{(1) \beta}, \cdots$ are orthogonal to each other. We shall investigate in the next section the structure of the transformations of $h$ operating on the irreducible invariant subspace $E^{(1) *}$ containing $E^{(1)}$.

2. The manifold $R^{*}$ and its holonomy group $H$. Suppose that the holonomy group $h$ of a complete Riemannian manifold $M_{n}$ is reducible to $r$ - and $(n-r)$-dimensional parts. Then there exist an $r$ dimensional parallel plane field and an $(n-r)$ dimensional parallel plane field orthogonal to each other. Let $K$ be an arbitrary curve in $M_{n}$. We denote its initial point by $P$ and its terminal point by $Q$. We take orthogonal repères $\left[e_{1}, \cdots, e_{n}\right]_{P}$ and $\left[e_{1}, \cdots, e_{n}\right]_{Q}$ so that their first $r$ vectors $\left[e_{1}, \cdots, e_{r}\right]_{P}$ and $\left[e_{1}, \cdots, e_{r}\right]_{Q}$ are contained in the $r$-dimensional planes of the first field at $P$ and $Q$ respectively and the remaining $(n-r)$ vectors $\left[e_{r+1}, \cdots, e_{n}\right]_{P}$ and $\left[e_{r+1}, \cdots, e_{n}\right]_{Q}$ 
are contained in the $(n-r)$-dimensional planes of the other field at $P$ and $Q$ respectively. By developing the curve $K$ in the tangent space $E_{n}(P)$ with respect to the Euclidean connection of the space, we find a unique image of the point $Q$ and a unique image $\left[e_{1}^{*}, \cdots, e_{n}^{*}\right]$ of the repère $\left[e_{1}, \cdots, e_{n}\right]_{\ell}$. Then the transformation which takes $\left[e_{1}, \cdots, e_{n}\right]_{P}$ to $\left[e_{1}^{*}, \cdots, e_{1}^{*}\right]$ can be expressed by a matrix $T\left(M_{n}, K\right)$ of the type

$$
\left(\begin{array}{lll}
A & 0 & \alpha \\
0 & B & \beta
\end{array}\right)
$$

where $A$ and $B$ are. $(r, r)$ and $(n-r, n-r)$ orthogonal matrices respectively and $\alpha$ and $\beta$ are $(r, 1)$ and $(n-r, 1)$ matrices respectively. Let us denote by $t\left(M_{n}, K\right)$ the matrix $(A, \alpha)$. The set of all transformations of the type $(A, \alpha)$ constitutes a group.

Let $K=\operatorname{arc} P Q$ be a sufficiently short curve of class $D^{\prime}$ so that it is contained in a so-called "reduced coordinate neighborhood" such that

$$
\begin{gathered}
d s^{2}=g_{a b}\left(x^{c}\right) d x^{a} d x^{b}+g_{p q}\left(x^{r}\right) d x^{p} d x^{q}, \\
a, b, c=1,2, \cdots, r, \\
p, q, r=r+1, \cdots, n .
\end{gathered}
$$

We denote $r$-dimensional totally geodesic submanifolds belonging to the family $x^{p}=$ const. by $R$ and $s-(=n-r)$ dimensional totally geodesic submanifolds belonging to the family $x^{a}=$ const. by $S$, and the $R$ - and $S$-submanifolds which pass through a point $P$ by $R_{P}$ and $S_{P}$.

We can project $K$ on $R_{P}$ by using the reduced coordinate neighborhood. We denote the projection by $\pi$ and denote the image of $K$ by $K^{\prime}=\pi(K)$. The repère $\left[e_{1}, \cdots, e_{r}\right]_{Q}$ impresses a repère $\left[e_{1}^{\prime}, \cdots, e_{r}^{\prime}\right]_{Q}$ at the image $Q^{\prime}=\pi(Q)$ on $R_{P}$. By developing the curve $K^{\prime}$ in the tangent space $E_{r}(P)$ of $R_{P}$ with respect to the induced Euclidean connection of the space, we find a unique image $\left[e_{1}^{\prime *}, \cdots, e_{r}^{\prime *}\right]$ of the repère $\left[e_{1}^{\prime}, \cdots, e_{r}^{\prime}\right]_{Q}$. Let us denote the transformation which takes $\left[e_{1}, \cdots, e_{r}\right]_{P}$ to $\left[e_{1}^{\prime *}, \cdots, e_{r}^{\prime *}\right]$ by $T\left(R_{P}, K^{\prime}\right)$. Then

Lemma 1. $t\left(M_{n}, K\right)=T\left(R_{P}, K^{\prime}\right)$.

If we write down the equations of definition of the Euclidean connection using a reduced coordinate neighborhood which contains $K$ we can easily see the truth of our assertion, for in this case the equations of definition of the connection separate into two parts corresponding to $R$ - and $S$-submanifolds.

Let $R_{a}$ and $R_{b}$ be two $R$-submanifolds and assume that $P \in R_{a}$ and $Q \in R_{b}$ lie on the same $S$-submanifold. By a $R$-neighborhood of $P$ we mean a neighborhood of $P$ in $R_{P}$. Then there exists an isometry $\chi$ between some $R$ neighborhoods $V(P)$ on $R_{a}$ and $V(Q)$ on $R_{b}$ such that corresponding points on $R_{a}$ and $R_{b}$ lie on the same $S$-submanifolds.

To prove this we connect $P$ and $Q$ by a geodesic $[P Q]$ in the $S$-manifold in which $P$ and $Q$ are. Divide $[P Q]$ so fine that if we denote the dividing 
points by $P=z_{0}, z_{1}, \cdots, z_{k}=Q$, the reduced neighborhood $U\left(z_{i}\right)$ of $z_{i}$ contains $z_{i+1}$. Then it is clear that there arises a sequence of isometries between suitable $R$-neighborhoods $V\left(z_{i}\right) \subset U\left(z_{i}\right) \cap R_{\boldsymbol{s}_{i}}(i=0,1, \cdots, k)$.

In the next place, let $L$ be a curve on $R_{a}$ passing through $P$. Then we can prolong the isometry $\chi$ of $V(P)$ and $V(Q)$ along $L$ on $R_{a}$ and its image on $R_{b}$, a point on $R_{a}$ and its image on $R_{b}$ lying always on the same $S$-submanifold.

To prove this we first take a point $P_{1}$ on the connected component of $P_{0} \equiv P$ on $V(P) \cap L$ and draw its image curve and denote the image of $P_{1}$ by $Q_{1}$. Draw the geodesic segment $\left[P_{1} Q_{1}\right]$ near $[P Q]$ and starting from $\left[P_{1} Q_{1}\right]$ we can construct an isometry between some $R$-neighborhoods $V\left(P_{1}\right)$ on $R_{a}$ and $V\left(Q_{1}\right)$ on $R_{b}$. It is evident that on $V(P) \cap V\left(P_{1}\right)$ and $V(Q) \cap V\left(Q_{1}\right)$ both isometries are identical and hence we can prolong the isometry $V(P) \rightleftarrows V(Q)$ to the isometry $V(P) \cup V\left(P_{1}\right) \rightleftarrows V(Q) \cup V\left(Q_{1}\right)$. Repeating this reasoning we can easily see that our assertion is true because we can choose a sequence of new neighborhoods so that the diameters of the new neighborhoods which arise successively by analogous construction have a positive lower bound. We denote the prolonged isometry also by $\chi$.

Let us denote the image of $L$ on $R_{b}$ by $M$. We impress the repère $\left[e_{1}, \cdots, e_{r}\right]_{P}$, at the initial point $P$ of $L$ spanning the $r$-dimensional tangent plane of $R_{a}$ at $P$ to the initial point $Q$ of $M$ and likewise impress the repère $\left[e_{1}, \cdots, e_{r}\right]_{P^{\prime}}$ at the terminal point $P^{\prime}$ of $L$ spanning the $r$-dimensional tangent plane of $R_{a}$ at $P^{\prime}$ to the terminal point $Q^{\prime}$ of $M$. Then

LEMMA 2. $T\left(R_{a}, L\right)=T\left(R_{b}, M\right)$.

Now let us consider a closed curve $C$ passing through the base point $O$ of the holonomy group. We divide the curve $C$ by $m$ points $O \equiv P_{0}, P_{1}, \cdots$, $P_{m-1}, P_{m} \equiv O$ so fine that the subarc $P_{\lambda} P_{\lambda+1}$ is contained in a reduced coordinate neighborhood $U\left(P_{\lambda}\right)$ for every $\lambda(\lambda=0,1, \cdots, m-1)$. We take at $P_{0}=P_{m}$ and $P_{1}, \cdots, P_{m-1}$ repères so that their first $r$ vectors $\left[e_{1}, \cdots, e_{r}\right]$ span $r$-dimensional tangent planes at them. And we consider the development of $C$ in the tangent space $E_{n}\left(P_{0}\right)$. Then, we get the following relation.

Lemma 3. $t\left(M_{n}, C\right)=T\left(R_{P_{0}}, C^{\prime}\right)$, where $C^{\prime}$ is the continuous projection of $C$ by $\pi$ such that $\pi\left(P_{0}\right)=P_{0}$.

Proof. First we get by virtue of Lemma 1

$$
t\left(M_{n}, C\right)=T\left(R_{P_{n-1}}, \operatorname{arc} P_{m-1} P_{m}^{\prime}\right) t\left(M_{n}, \operatorname{arc} P_{0} P_{m-1} \text { of } C\right),
$$

where $\operatorname{arc} P_{m-1} P_{m}^{\prime}$ is the image of the $\operatorname{arc} P_{m-1} P_{m}$ by the projection $\pi$. The right-hand side of the last equation is, by virtue of Lemmas 1 and 2, equal to the following transformation:

$$
T\left(R_{P_{m-2}}, \text { arc } P_{m-2} P_{m}^{\prime \prime}\right) t\left(M_{n}, \text { arc } P_{0} P_{m-2} \text { of } C\right),
$$

where the $\operatorname{arc} P_{m-2} P_{m}^{\prime \prime}$ is the image of $\left(\operatorname{arc} P_{m-2} P_{m-1}\right.$ of $\left.C\right)+\operatorname{arc} P_{m-2} P_{m-1}^{\prime}$ by 
the projection $\pi$ and isometry $\chi$ on $R_{P_{m-1}}$. Iterating this process we can see that Lemma 3 is true.

We are now going to assume a hypothesis $\left({ }^{2}\right)$.

Hypothesis W. There exists in $M_{n}$ a point $O$ such that each point of the submanifold $R_{0}$ has an $R$-neighborhood which meets at most once with any $S$-submanifold.

When this hypothesis is satisfied, we take such a point $O$ as the base point of holonomy groups. Then, for any $S$-submanifold, the intersection $S \cap R_{0}$ is a discrete set of points. We say that any two points of this set are congruent to each other. Then a sufficiently small $R$-neighborhood of a point on $R_{0}$ is isometric with corresponding $R$-neighborhoods of its congruent points. Hence if we identify congruent points on $R_{0}$, there arises a manifold $R^{*}$ such that $R_{0}$ is a covering manifold of $R^{*}$. As $M_{n}$ is assumed to be complete, $R_{0}$ and $R^{*}$ are also complete. The terminal point $O^{\prime}$ of the curve $C^{\prime}$ does not in general coincide with the point $O$, except in the case when $C$ is homotopic to zero. However, by the construction, $O^{\prime}$ is congruent to $O$. Hence the image $C^{*}$ of $C^{\prime}$ on $R^{*}$ is a closed curve passing through $O^{*}$ (image of $O$ ) on $R^{*}$. As $R_{0}$ and $R^{*}$ correspond locally isometrically we can easily see that

$$
T\left(R_{0}, C^{\prime}\right)=T\left(R^{*}, C^{*}\right) \in H\left(R^{*}\right),
$$

where $H\left(R^{*}\right)$ means the holonomy group of $R^{*}$.

Conversely, let us consider a closed curve of class $D^{\prime}$ passing through the base point $O^{*}$ and the transformation $T\left(C^{*}\right)$ of the holonomy group $H\left(R^{*}\right)$ associated with $C^{*}$. As $R_{0}$ is a covering manifold of $R^{*}$ we can construct the curve $C^{\prime}$ which issues from the point $O$ over $O^{*}$ and lies over $C^{*}$. The terminal point $O^{\prime}$ of $C^{\prime}$ is a point which is congruent to $O$. We can easily see that

$$
T\left(C^{*}\right)=T\left(R_{0}, C^{\prime}\right),
$$

provided that the repères $\left[e_{1}, \cdots, e_{r}\right]_{o}$ and $\left[e_{1}, \cdots, e_{r}\right]_{o^{\prime}}$ at the initial and terminal points of $C^{\prime}$ are those which lie over the repère at the point $O^{*}$.

Now, as $O$ and $O^{\prime}$ lie on the same submanifold $S_{0}$ we can connect $O^{\prime}$ with $O$ by a curve $C^{\prime \prime}$ of class $D^{\prime}$ on $S_{0}$. If we consider the product curve $C^{\prime} C^{\prime \prime}$ as a curve in $M_{n}$, the continuous projection of $C^{\prime} C^{\prime \prime}$ into $R_{0}$ is easily seen to be $C^{\prime}$. The repère $\left[e_{1}, \cdots, e_{r}\right]_{0^{\prime}}$ we mentioned above is also the image of $\left[e_{1}, \cdots\right.$, $\left.e_{r}\right]_{o}$ by the projection. Hence we can see that

$$
T\left(R_{0}, C^{\prime}\right)=t\left(M_{n}, C^{\prime} C^{\prime \prime}\right) .
$$

Consequently, we get the following

ThEOREM 2. Let $M_{n}$ be a complete Riemannian manifold whose holonomy group $h$ decomposes in r-dimensional and $(n-r)$-dimensional parts and satisfies the hypothesis $\mathrm{W}$. We take a point $O$ satisfying the hypothesis $\mathrm{W}$ and construct

(2) A. G. Walker, The fibering of Riemannian manifolds, Proc. London Math. Soc. (3) vol. 3 (1953) pp. 1-19. 
the submanifold $R_{0}$ and the manifold $R^{*}$ which arises by identification of congruent points of $R_{0}$. Then the group which consists of all transformations $t\left(M_{n}, C\right)$ is the same as the holonomy group $H\left(R^{*}\right)$ of the manifold $R^{*}$, the base point $O^{*}$ and the repere at $O^{*}$ on $R^{*}$ being naturally impressed from $M_{n}$.

Suppose that the restricted holonomy group $h^{0}$ of $M_{n}$ decomposes and fixes $r$ vectors (we do not assume that there are no other invariant vectors), and that these $r$ vectors span an $r$-dimensional plane invariant under the holonomy group $h$. Then there exist a parallel field of $r$-dimensional planes in $M_{n}$ and $(n-r)$-parameter family of $r$-dimensional totally geodesic submanifolds $R$. Each of these submanifolds $R$ is an Euclidean space form, in other words, a complete manifold with locally flat Riemannian metric. Hence the manifold $R^{*}$ is also a Euclidean space form. However, as is known, "the universal covering manifold of any Euclidean space form of $n$ dimensions is the $n$-dimensional Euclidean space $E_{n}$ and the holonomy group $H$ of it coincides with the group of covering transformations on $E_{n}$ ". Hence, $H\left(R^{*}\right)$ is nothing but a discrete group of congruent transformations without fixed points of $E_{n}$. Accordingly we get, by virtue of Theorem 2, the following

THEOREM 3. Suppose that the holonomy group $h^{0}$ of a complete Riemannian manifold $M_{n}$ decomposes and fixes $r$ vectors and that these $r$ vectors span an $r$-dimensional plane invariant under the holonomy group $h$. If it moreover satisfies the hypothesis $\mathrm{W}$, the r-dimensional part corresponding to the invariant $r$ dimensional plane of the holonomy group $H\left(M_{n}\right)$ is a discrete group of congruent transformations without fixed points of $E_{n}$.

REMARK. We can easily see from the fact " . . " cited above that a (nonrestricted) homogeneous holonomy group $h$ is not always closed in the orthogonal group $O(n)$. For example, consider the cyclic group generated by the following transformation of $E_{n}$

$$
\begin{aligned}
& x_{1}^{\prime}=\cos \alpha x_{1}-\sin \alpha x_{2}, \\
& x_{2}^{\prime}=\sin \alpha x_{1}+\cos \alpha x_{2}, \\
& x_{3}^{\prime}=x_{3}+1 .
\end{aligned}
$$

The factor space of $E_{n}$ by this group has obviously the desired property.

3. A theorem on the group $H^{0}$.

Theorem $4\left({ }^{3}\right)$. Let $M_{n}$ be an irreducible Riemannian manifold. Then the holonomy group $H^{0}$

(i) either contains all translations of Euclidean space $E_{n}$

(ii) or it fixes a point in $E_{n}$ (in other words, it is a subgroup of the rotation group $O^{+}(n)$ with a center at the fixed point).

(3) We owe this theorem to A. Borel. But for the sake of completeness we shall write our proof here. 
Proof. We shall indicate an element of $H^{0}$ considered as a topological group by $g$ and the motion associated with $g$ by

$$
T(g): x_{i}^{\prime}=a_{i j}(g) x_{j}+a_{i}(g) .
$$

Then all the transformations $T(g)$ constitute $H^{0}$. Of course, the matrices

$$
A(g)=\left(a_{i j}(g)\right)
$$

are nothing but the coefficient matrices of transformations of $h^{0}$ and this set is irreducible by our assumption.

Now let us consider the representation $\Gamma: g \rightarrow A(g)$ and denote the kernel of $\Gamma$ by $K$. Then $K$ is the totality of elements of $H^{0}$ such that $A(g)=E$, hence $K$ is the subgroup of $H^{0}$ consisting of all translations of $H^{0}$. We shall classify two cases; the first is the case where $K$ is nondiscrete and the second is the case where $K$ is discrete.

(i) The case where $K$ is a nondiscrete group. As $K$ is a closed subgroup of $H^{0}, K$ is a Lie group. Hence $K$ contains at least a one parameter group $K_{1}$ of translations as its subgroup. Let us denote it by

$$
\Lambda_{t}: x_{i}^{\prime}=x_{i}+\lambda_{i} t
$$

where $\lambda_{i}$ are constant such that at least one of them is not equal to zero. Now denoting an arbitrary element of $H^{0}$ by $T(g)$ we can easily verify that $T(g) \Lambda_{t} T(g)^{-1}$ is a translation and its equation is given by

$$
x_{i}^{\prime}=x_{i}+a_{i h}(g) \lambda_{h} t .
$$

As the set of matrices $\left(a_{i j}(g)\right)$ is irreducible, we see immediately that $K$ contains $n$ linearly independent translations. Hence $K$ contains all translations of $E_{n}$.

(ii) The case where $K$ is a discrete group. As $K$ is the kernel of the representation $\Gamma, K$ is a normal subgroup of $H^{0}$. Hence, by virtue of the theorem ( $\left.{ }^{4}\right)$ to the effect that every discrete normal subgroup of a connected topological group is a central normal subgroup of this group, $K$ is contained in the center of $H^{0}$. Accordingly, if we assume that $T(g)$ and $\Lambda: x_{i}^{\prime}=x_{i}+\lambda_{i}$ are transformations belonging to $H^{0}$ and $K$ respectively, then $T(g) \Lambda T(g)^{-1}$ must coincide with $\Lambda$. Hence, we can see that the equation

$$
\lambda_{i}=a_{i k}(g) \lambda_{k}
$$

must hold for every $g \in H^{0}$ and fixed $\lambda_{i}$. If there is one $\lambda$ which is not equal to zero among $\lambda_{i}$, the last equation shows that there exists at least an invariant direction under $h^{0}$ which contradicts the fact that $h^{0}$ is irreducible by our assumption. Accordingly, every $\lambda_{i}$ must vanish and hence $K$ consists only of the identity. Consequently, we can conclude that the representation $\Gamma$ is faithful, in other words there exists an isomorphism $H^{0} \cong h^{0}$.

(4) Pontrjagin, Topological groups, p. 77. 
By virtue of a theorem of Borel-Lichnerowicz $\left(^{(5)}\right.$, the group $h^{0}$ is a closed subgroup of the compact orthogonal group $O^{+}(n)$ and hence $h^{0}$ is compact. As $H^{0} \cong h^{0}, H^{0}$ is also compact. Accordingly, we can introduce in the group manifold of $H^{0}$ the Haar measure. Denoting the total measure of the group manifold by $\omega$, we put

$$
\frac{1}{\omega} \int a_{i}(g) d g=c_{i}
$$

In the last and the following equations we assume that the integrals are extended over the whole group manifold. Then we can easily see that

$$
a_{i k}(g) c_{k}+a_{i}(g)=\frac{1}{\omega} \int\left(a_{i k}(g) a_{k}(h)+a_{i}(g)\right) d h .
$$

As the integrand of the right-nand side of the last equation is equal to $a_{i}(h g)$, we get

$$
a_{i k}(g) c_{k}+a_{i}(g)=\frac{1}{\omega} \int a_{i}(h g) d h .
$$

Since the Haar measure over the compact group is two-sided invariant, the right-hand side is equal to

$$
\frac{1}{\omega} \int a_{i}(h) d h=c_{i}
$$

Consequently, $c_{i}$ is the fixed point under the group $H^{0}$. Thus our theorem is completely proved.

\section{A theorem on complete Riemannian manifolds.}

TheOREM 5. Let $M_{n}$ be a complete Riemannian manifold. If the holonomy group $H^{0}$ fixes a point, then $M_{n}$ is an Euclidean space form.

Proof. We shall prove the theorem under the assumption that $M_{n}$ is simply connected. However, if this is done, the general case follows immediately. For, as the holonomy group $\widetilde{H}=\widetilde{H}^{0}$ of the universal covering manifold $\tilde{M}_{n}$ of $M_{n}$ with naturally induced matric from $M_{n}$ coincides with $H^{0}, \tilde{M}_{n}$ is an Euclidean space form by hypothesis and hence $M_{n}$ itself is everywhere locally Euclidean.

Let us denote the base point of the holonomy group by $O$ and denote the fixed point in the tangent space $E_{n}(O)$ by $P_{0}{ }^{*}$. Then, we can draw a geodesic segment arc $O P_{0}$ in $M_{n}$ such that its development in $E_{n}(O)$ coincides with the straight line $O P_{0}^{*}$ by virtue of the completeness assumption. We shall consider a normal coordinate system with center $P_{0}$ and denote it by $\bar{x}$ and the

(5) A. Borel and A. Lichnerowicz, loc. cit. 
coordinate neighborhood by $U$. It is well known that

$$
\left\{\begin{array}{c}
\bar{i} \\
j k
\end{array}\right\} \bar{x}^{i} \bar{x}^{k}=0
$$

holds good in $U$.

Now let us consider a point $P-\bar{x}^{i} e_{i}$ at every tangent space $E_{n}(P)(P \in U)$ on a geodesic $\bar{x}^{i}=a^{i}$ through $P_{0}$, where we assume that $e_{i}$ is the natural repère at that point. Then, by virtue of (4) we get

$$
\frac{d}{d s}\left(P-\bar{x}^{i} e_{i}\right)=\left(\frac{d \bar{x}^{i}}{d s}-\frac{d \bar{x}^{i}}{d s}-\bar{x}^{j}\left\{\begin{array}{c}
\bar{i} \\
j k
\end{array}\right\} a^{k}\right) e_{i}=0 .
$$

Hence, the point $P-\bar{x}^{i} e_{i} \in E_{n}(P)$ at each point $P$ of the geodesic $\bar{x}^{i}=a^{i s}$ concides when we develop these tangent spaces along the geodesic. If we consider the case $\bar{x}^{i}=0$ we can see that the point $P-\bar{x}^{i} e_{i}$ coincides with the invariant point $P_{0}^{*}$ of the holonomy group $H^{0}$. Hence $P-\bar{x}^{i} e_{i}$ in $E_{n}(P)$ is the point which is transplanted from $P_{0}^{*}$ by the connection of the manifold $M_{n}$, and it does not depend upon the curves which combine $P_{0}$ to $P$.

Let us now consider another curve $C$ through $P$ and consider the derivative of $P-\bar{x}^{i} e_{i}$ with respect to this curve. As $P-\bar{x}^{i} e_{i}$ is a covariant constant point field, we get $(d / d s)\left(P-\bar{x}^{i} e_{i}\right)=0$, which reduces to

$$
\left\{\begin{array}{c}
\bar{i} \\
j k
\end{array}\right\} \bar{x}^{j} \frac{d \bar{x}^{k}}{d s} e_{i}=0 .
$$

As the curve $C$ may have any direction at $P$, we get from the last equation the following relation:

$$
\left\{\begin{array}{c}
\bar{i} \\
j k
\end{array}\right\} \bar{x}^{j}=0
$$

From the last relation we get easily

$$
\left(\partial \bar{g}_{j k} / \partial \bar{x}^{i}\right) \bar{x}^{i}=0,
$$

which shows that $\bar{g}_{j k}(\bar{x})$ 's are homogeneous functions of degree 0 with respect to $x^{i(8)}$. Hence, as $P_{0}$ is a regular point of the manifold $M_{n}$, we see that $\bar{g}_{j k}(\bar{x})=\bar{g}_{j k}(0)$ in the neighborhood $U$. Accordingly, in the domain $U$ our manifold is locally Euclidean.

In the next place we shall show that any two geodesic rays which issue from $O$ do not intersect any more. By hypothesis $M_{n}$ is simply connected. Hence the point $P_{0}^{*}$ can be transplanted uniquely on every tangent space $E_{n}(P)$ $\left(P \in M_{n}\right)$ by development irrespective to curves which bind $P_{0}$ to $P$. It is

(6) S. Tachibana, On the normal coordinate of Riemann space, whose holonomy group fixes a point, Tôhoku Math. J. (2) vol. 1 (1949) pp. 26-30. 
evident that the transplanted point does not coincide with $P$ unless $P$ coincides with $P_{0}$. Now, let us assume that there exist two geodesic rays which intersect at $Q(\neq P)$ and denote them by $g_{1}$ and $g_{2}$. If we consider the points $R_{1}^{*}, R_{2}{ }^{*}$ on $E_{n}(Q)$ which lie on the tangents of $g_{1}$ and $g_{2}$ at $Q$ such that $Q R_{\lambda}{ }^{*}$ $=$ the length of arc $Q P_{0}$ along $g_{\lambda}(\lambda=1,2)$, then $R_{1}^{*}, R_{2}^{*}$ are the transplanted points of $P_{0}^{*}$ along $g_{\lambda}$. This contradicts the fact that $P_{0}^{*}$ is transplanted uniquely irrespective to curves which bind $P_{0}$ to $Q$. Accordingly, any two geodesic rays which issue from $P_{0}$ do not intersect any more. In other words, geodesic rays which issue from $P_{0}$ constitute a geodesic field in the large.

Now let us denote by $K_{l}$ the inner domain of the geodesic hypersphere of radius $l$ and with center $P_{0}$ and by $\partial K_{l}$ its boundary. We take $l$ so large that every point $P \in K_{l}$ has a locally flat neighborhood but some points on $\partial K_{l}$ do not have such property. If $l=\infty$, then our theorem is proved, so we assume that $l$ is finite and $Q \in \partial K_{l}$ is one of the points which do not have the above stated property.

As the group $H^{0}$ fixes the point $P_{0}^{*}$, there exists a neighborhood $V$ of $Q$ such that the line element $d s^{2}$ in $V$ can be written as $\left({ }^{7}\right)$

$$
d s^{2}=\left(x^{n}\right)^{2} g_{a b}\left(x^{c}\right) d x^{a} d x^{b}+\left(d x^{n}\right)^{2} \quad a, b, c=1,2, \cdots, n-1 .
$$

If a point $R \in V$ has the coordinates $\left(x^{a}, x^{n}\right)$, then $x^{n}=$ the length of the geodesic segment $P_{0} R$ and the geodesic hyperspheres $x^{n}=$ const. are umbilical hypersurfaces too. However, when $x^{n}<l$ the line element is locally flat by assumption, hence it is also locally flat for $x^{n} \geqq l$. Hence the line element is locally flat in $V$. This contradicts the fact that $Q$ has no locally flat neighborhood. Accordingly $l=\infty$ i.e. $M_{\mathrm{n}}$ is locally Euclidean everywhere. Q.E.D.

COROLLARY 1. Let $M_{n}$ be a complete Riemannian manifold which is irreducible (with respect to the holonomy group $h^{0}$ ). Then the holonomy group $H^{0}$ contains all translations of the Euclidean space $E_{n}$.

COROLlary 2. Let $M_{n}$ be a complete Riemannian manifold. Then the holonomy group $H^{0}$ is a closed subgroup of the group of motions.

COROLlary 3. Let $M_{n}$ be a complete and simply connected Riemannian manifold. If its nonhomogeneous holonomy group $H^{0}$ fixes an $r$-dimensional linear subspace $E_{r}(0<r<n)$ of $E_{n}$, then

$$
V_{r}=V_{x} \times E_{n-r}
$$

The INSTITUTE For AdVANCEd Study, Princeton, N. J.

(7) S. Sasaki, On the structure of Riemannian spaces whose holonomy groups fix a direction or a point, Journ. Physico Math. Soc. Japan vol. 16 (1942) pp. 193-200 (in Japanese). 